\title{
GR@PPA 2.9: radiation matching for simulating photon production processes in hadron collisions
}

\author{
Shigeru Odaka*, Yoshimasa Kurihara \\ High Energy Accelerator Research Organization (KEK) \\ 1-1 Oho, Tsukuba, Ibaraki 305-0801, Japan
}

\begin{abstract}
We release an event generator package, GR@PPA 2.9, for simulating the direct (single) photon and diphoton (double photon) production in hadron collisions. The included programs were used in our previous studies, in which we have explicitly shown large contributions from parton-associated processes. The programs consistently combine simulations based on matrix elements with parton-shower simulations that reproduce the multiple parton radiation and quark fragmentation to photons. The matrix elements include associated parton production processes up to two partons. We provide instructions for the installation and execution of the programs in this article. The practical performance is also presented.
\end{abstract}

Keywords: GRACE; Hadron collision; Photon production; Event generator; Radiation matching

\section{PROGRAM SUMMARY}

Manuscript Title: GR@PPA 2.9: radiation matching for simulating photon production processes in hadron collisions

Authors: S. Odaka, Y. Kurihara

Program Title: GR@PPA 2.9

Journal Reference:

Catalogue identifier:

Licensing provisions: none

Programming language: Fortran; with some included libraries coded in $\mathrm{C}$ and $\mathrm{C}++$

Computer: all

Operating system: any UNIX-like system

RAM: 4 Maga bytes

Supplementary material:

Keywords: GRACE; Hadron collision; Photon production; Event generator; Radiation matching

Classification: 11.2

External routines/libraries: bash and Perl for the setup, and CERNLIB, ROOT, LHAPDF 5, PYTHIA 6.4 according to the choice of users

Does the new version supersede the previous version?: No, this version supports processes not included in previous versions.

Nature of problem: It is necessary to include jet-associated processes up to two jets for realistic simulations of photon production processes in high-energy hadron collisions. Photons also need to be considered as radiation. An appropriate matching method has to be introduced for combining those processes having different jet and photon multiplicities and parton shower simulations.

Solution method: The limited leading-log (LLL) subtraction method has been extended to multi-jet processes and those including photon radiation. The final-state parton shower simulation has been improved to support QED photon radiation.

Reasons for the new version: New processes have been supported.

Summary of revisions: This program package provides event generators for realistic simulations of single and double photon production processes in high-energy hadron collisions. Users can consistently combine different

${ }^{*}$ Corresponding author.

E-mail address: shigeru.odaka@kek.jp 
jet- and photon-multiplicity processes up to two jets and two photons.

Restrictions:

Unusual features:

Running time: The CPU time consumption is strongly dependent on the process. An example is presented in the text for a sample program.

\section{Introduction}

High-energy isolated photon production is a clean signal in busy final states of hadron collisions and is considered to be suitable for probing the internal structure of the colliding hadrons [1,2], as such photons are expected to be produced via well-known Quantum Electrodynamic (QED) interactions of constituant partons (gluons and quarks), which is referred to as the direct photon production.

Despite such a prospect, this process has been a mystery for a long time. Theories, even the next-to-leading order (NLO) calculations in Quantum Chromodynamics (QCD), could not reproduce measurement results, and intensive studies and debates were made to solve the problem (see, for example, $[3,4]$ and the references therein). As higher energy collision data became available $[5,6,7,8]$, it has been revealed that the photon production in high transverse momentum $\left(p_{T}\right)$ regions $(\gtrsim 50$ $\mathrm{GeV}$ ) can be reasonably described by the NLO calculation [9], although discussions are yet to be conclusive for lower $p_{T}$ production.

From the discussions, it has been recognized that appropriate treatments of divergent collinear contributions in both QCD and QED, the multiple radiation in QCD, and the fragmentation in QED, are important. Possible problems in the definition of the isolation condition have also been addressed [10]. In addition, although it has not been emphasized explicitly, the NLO correction that includes the effect of associated two-parton production was found to be unreasonably large in the sense of perturbation. The correction amounts to more than $100 \%$ under typical measurement conditions $[9]^{1}$.

Along with the direct (single) photon production, diphoton (double photon) production is also an important process, especially in searches for new phenomena. Indeed, the diphoton production at the Large Hadron Collider (LHC) played an important role in the discovery of the Higgs boson [12, 13]. In the diphoton mode, the Higgs boson was observed as a small resonance on a large background from other interactions. Hence, the understanding of the non-resonant background is crucial for detailed studies on the discovered Higgs boson properties.

The diphoton production also shows a dramatic and mysterious behavior. First of all, the NLO correction is markedly large; it is more than $200 \%$ under typical measurement conditions [14]. This is predominantly caused by a very large contribution of quark-gluon $(q g)$ interactions that newly participate in this order. Furthermore, it was recently found that the next-to-next-to-leading order (NNLO) correction that includes the effects of associated two-parton production is also large, as in the direct photon production $[15,16]$. Hence, this order of correction is necessary to be included in order to realistically reproduce the non-resonant diphoton production $[17,18]$, although we still do not have any reasonable explanation on this large correction. Here, we note that the large NNLO correction is not predominantly caused by the emergence of new processes, e.g., $g g \rightarrow \gamma \gamma q \bar{q}$, but mainly originates from ordinary gluon-radiation corrections to one-parton production processes [15].

In this article, we describe a program package, GR@PPA 2.9, for simulating the direct photon and diphoton production in proton-proton $(p p)$ and proton-antiproton $(p \bar{p})$ collisions. The included event generator programs were used for the studies reported in our previous articles [19, 20,21], in which we confirmed large contributions from parton-associated processes. The predominance of gluon-radiation processes among two-parton processes was also found in the direct-photon production [21].

In GR@PPA 2.9, the event generation is based on leading order (LO) matrix elements (MEs) for the processes including associated parton production up to two partons. The programs for calculating the MEs were produced by using the GRACE system [22, 23]. The predominant collinear corrections

\footnotetext{
${ }^{1}$ Recently, the NNLO correction to the direct photon production was found to be moderate [11].
} 
of QCD, which lead to the multiple radiation of partons, are simulated by applying parton shower (PS) simulations to the generated events $[24,25,26,19]$. The simulation can proceed further to the hadron level with the help of external general-purpose event generators, such as PYTHIA [27].

The QCD corrections are involved in both ME-based event generation and PS. The matching between the two simulations is achieved using the limited leading-log (LLL) subtraction method [28, $24,19]$, in which the divergent leading-logarithmic (LL) components are numerically subtracted from squared MEs of radiative processes to make them finite. The LL components are the leading terms of PS. We achieve good matching by carefully arranging the PS kinematics and energy scale matching, to combine the events that are generated according to the MEs of different parton multiplicities ${ }^{2}$.

Along with the collinear divergence, we also need to consider the soft-gluon divergence to make the two-parton production processes finite. We have introduced a combined subtraction method for subtracting these divergences simultaneously [31]. A small correction is applied to the PS-applied lower parton-multiplicity events in order to compensate for this alteration in the subtraction.

In the measurements of photon production, we cannot completely avoid QED collinear divergences even if isolation requirements are imposed on the photons. We may miss the hadronic activities induced by the quark that radiated the detected photon, if the remaining quark momentum after the radiation is small. We apply the QED LLL subtraction to regularize such QED collinear divergences [19]. The PS simulation involves QED photon radiation for restoring the subtracted collinear components. In addition, very small $Q^{2}$ regions that the PS simulations do not cover are simulated on the basis of a fragmentation function (FF) [32]. This PS/FF simulation has the ability to enforce the radiation of a given number of energetic photons for efficient event generation [19, 21].

We can combine the events of processes involving associated partons in the final state up to two partons using the programs in GR@PPA 2.9. Therefore, the approximation order is the same as NLO in the direct photon production and NNLO in the diphoton production. However, finite components in loop corrections and next-to-leading logarithmic (NLL) components are missing. Accordingly, the overall normalization is not theoretically guaranteed. In contrast, transverse activities induced by the QCD multiple radiation, which are ignored in perturbative calculations, are reproduced by the PS in our simulation. We can finally obtain exclusive hadron-level event information. These features allow us to perform detailed studies on the acceptance, isolation conditions, and associated hadron-jet production which cannot be carried out using perturbative calculations.

The simulation method and the resultant physics performance have already been presented in previous articles $[19,20,21]$. We present only the execution instruction and practical performance of the programs in this article. The remainder of this article is organized as follows. The instructions for building the libraries and sample programs are provided in Section 2. Section 3 describes the control parameters that are newly introduced in this version. The practical performance of a sample program is presented in Section 4, and the descriptions are summarized in Section 5.

\section{Instruction}

\subsection{Distribution package}

The program package of GR@PPA 2.9 is distributed as a gzipped tar file named GR@PPA-2.9.tgz, which can be obtained from the GR@PPA Web page ${ }^{3}$. The compressed file can be expanded, for instance, by typing

tar zxf GR@PPA-2.9.tgz

on UNIX systems. When the file is expanded, users have a directory named GR@PPA-2.9 containing the following files and directories:

(miscellaneous files)

\footnotetext{
${ }^{2}$ Other approaches to the matching in photon production processes can be found in $[29,30]$.

${ }^{3}$ http://atlas.kek.jp/physics/nlo-wg/grappa.html\#GRAPPA2.9
} 
README : readme file describing how to use this package,

VERSION-2.9 : file to show the version number,

(files for setup)

config.input : file to specify the configuration for the setup,

Install : shell script for the installation,

config : shell script to configure the setup,

config.perl : Perl script called by config,

proc.list : process list, which is referred to in config.perl,

(GR@PPA framework)

grckinem : source files of the framework,

basesv5.1 : BASES 5.1 source package with some customization,

chanel : CHANEL source package to define the interaction model,

inc : directory containing common include files,

example : directory to be used for the setup of sample programs,

diagrams : directory containing PS files illustrating typical Feynman diagrams,

lib : directory to store object libraries; initially empty,

ffphoto : QED fragmentation function,

(process directories)

ajets : single-photon production processes,

aajets : double-photon production processes,

qcd : QCD-jet production processes,

handcode : handcoded ME programs.

The setup files and framework directories are almost identical to those in the GR@PPA 2.8 series [26]. The ffphoto directory has been added to simulate the small $Q^{2}$ photon radiation according to an FF [32]. All modifications to the framework routines are now backported to recent versions of the 2.8 series, e.g., GR@PPA 2.8.6.2.

The process directories are entirely different from those in the 2.8 series. The directories ajets and aajets contain ME calculation programs for single and double photon production processes, respectively. The qcd directory contains the programs for QCD 2-jet production, in which photons can be produced if energetic photons are radiated in the PS simulation. The handcode directory contains handcoded ME programs. Programs for the loop-mediated $g g \rightarrow \gamma \gamma$ process are provided in the present version.

\subsection{Installation}

The installation procedure is also identical to that for the GR@PPA 2.8 series. Move into the expanded distribution package, and appropriately edit the file config.input to specify the parton distribution function (PDF) library, compilation tools, and the location of external libraries.

For the PDF library, users have to select one of the following options: CTEQL1, LHAPDF, or PDFLIB, although the option PDFLIB is now obsolete because programs built with this option refer to the old PDFLIB library in CERNLIB. The programs built with CTEQL1 use the CTEQL1 PDF [33] contained in this package. This choice is convenient for a quick check of the program. The option LHAPDF should be selected for intensive studies. The programs built with this option refer to an LHAPDF library $^{4}[34]$. If users select this option, they have to prepare an external LHAPDF library and specify its location in config.input.

External libraries are necessary for building sample programs. Although they can be modified later, it is recommended to specify them in config. input to ensure the consistency. Here, unnecessary libraries are allowed to be left unspecified.

The libraries are compiled and installed in the lib directory by executing

\footnotetext{
${ }^{4}$ We have been using the LHAPDF version 5 for our studies. The compatibility with the version 6 has not been tested.
} 
at the top of the expanded distribution package. It may be better to separately execute the two commands, ./config and make, when certain problems are encountered. More details about the installation can be found in the GR@PPA-2.8 manual [26].

\subsection{Sample programs}

\subsubsection{Built-in samples}

Sample programs are provided under the ajets/example and aajets/example directories for a quick check of the installation. Programs using both HBOOK and RBOOK are provided as in the GR@PPA-2.8 distributions. Users can build and execute the program by invoking the commands

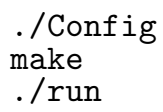

in one of the sample program directories. The sample programs produce an event file using the LhaExt utility $^{5}$. The version 2.0 of LhaExt is implemented in GR@PPA 2.9 and recent versions of GR@PPA 2.8 , in which the XML-based LHEF format of event data [35] is supported.

The event data can be further processed down to the hadron level with the help of PYTHIA 6.4 [27] by executing the program provided under the pythia directory in each sample. After the hadronization, the final angular and momentum selection is applied and the isolation requirement is imposed to the photons in these programs. The parameters in PYTHIA 6.4 are left unchanged from the default in the sample programs, except for PARP $(67)=1.0$ and $\operatorname{PARP}(71)=1.0$, which are explicitly specified in the source program Pythia.f. This setting should be applied when the default "old" PS is used in PYTHIA. Users can build and execute the sample programs by invoking the above three commands again in the pythia directory.

Contrary to heavy particle production, in which no-cut event generation is possible, strict angular and momentum constraints are necessary to be imposed in photon production processes. The constraints need to be applied at several stages of the simulation in order to optimize the event generation efficiency. In the sample programs, constraints are imposed at the generation of parton-level hardinteraction events, after applying the PS simulations, and finally after completing the hadronization. Since only the final selection at the hadron level is physically meaningful, we need to confirm that the selected sample is not biased by the constraints of earlier stages.

In order to make the above confirmation possible, the sample programs are arranged in such a way that the initial hard-interaction information can be added to the event information that is passed to the PYTHIA application program. The added information is separated from the ordinary event information in the UPEVNT subroutine attached to Pythia.f, so that the information can be referred in other parts of the program. The earlier constraints are safe in the final event sample if the distributions of the used quantities vanish before reaching the applied cuts.

\subsubsection{Matched direct photon and diphoton + two jets}

Program sets for simulating the direct photon and diphoton production in $7-\mathrm{TeV}$ proton-proton collisions can be obtained from the GR@PPA Web page. Associated jet production is included up to two jets in these samples. Here, jet refers to the produced parton because energetic parton production is considered to be observed as the production of a hadron jet. The programs were used to simulate the ATLAS measurements in previous articles $[20,21]$. In these external samples, the events of different photon and parton multiplicities are generated separately. Such a procedure is convenient because the program setting and required CPU time are markedly different between the subprocesses.

As these programs are separated from the distribution package, Makefile and the usr directory have to be manually copied from one of the properly-built built-in sample programs described above. The file Ihapdf_setup.sh and the symbolic link PDFsets have also to be copied when LHAPDF is

\footnotetext{
${ }^{5}$ http://atlas.kek.jp/physics/nlo-wg/grappa.html\#LhaExt
} 
Table 1: New or extended parameters to be defined in UPINIT. "D" denotes the default setting.

\begin{tabular}{ll}
\hline Parameter & Description \\
\hline labcut & switch for activating the LabCut framework. $(\mathrm{D}=0)$ \\
& $=0:$ deavtivate. \\
& $=1:$ activate. \\
ishwfin & switch for the final-state PS. $(\mathrm{D}=0)$ \\
& $=0:$ no PS is applied. \\
& $=1:$ QCDPSf is applied. \\
& $=2:$ QCDPSf including QED FSR is applied. \\
& $=3:$ QCDPSf with forced QED FSR is applied. \\
& switch for activating the soft-gluon correction. $(\mathrm{D}=0)$ \\
& $=0:$ deactivate. \\
& $=1:$ activate.
\end{tabular}

selected for the PDF library. If these files and directories are copied correctly, the programs can be built by simply invoking the make command and can be executed by typing ./run. These samples contain a PYTHIA application program in each subprocess directory for checking the generation and selection conditions in the GR@PPA event generation.

Another PYTHIA application program is provided outside the event generation directories in these samples. These programs have a function of mixing separately generated event data. The mixing is carried out randomly in proportion to the integrated cross section of each subprocess, so that the obtained results can be directly compared with measurement results. A sample Makefile is provided because the I/O scheme is unusual in these programs. The event files to be read are explicitly specified in the subroutine upinit included in Pythia.f.

The execution results of these sample programs with the use of the CTEQ6L1 PDF are provided under the results directories. The BASES result files and log files are provided for event generation programs, and the log files are provided for PYTHIA application programs. These sample results will be useful for the users to confirm the execution.

\section{Control parameters}

As can seen in the sample programs, users are allowed to modify the programs in four files, grappa.f, upinit.F, upevnt.f, and grcpar.F for the GR@PPA event generation. Among them, upevnt.f must be less attractive because it simply calls the steering routine of GR@PPA for generating one event. The generation conditions are specified in upinit.F and grcpar.F. The user analyses and data I/O are controlled in the main program in grappa.f. In the following subsections, we describe how various execution conditions that have been introduced after the release of GR@PPA 2.8 can be specified in these files. Refer to the GR@PPA 2.8 manual [26] for the other functions of GR@PPA.

\section{1. upinit.F}

The file upinit.F contains only one subroutine UPINIT, which is called only once at the beginning of the event generation. Thus, the conditions that are valid through the execution are defined in this routine. New or extended parameters that are supported after the release of GR@PPA 2.8 are summarized in Table 1.

The LabCut framework is activated by setting labcut $=1$ in all the sample programs. In this framework, PS simulations are applied before passing the differential cross section value to the integration and event generation utility, BASES/SPRING [36, 37]. The event weight that is evaluated after the application of PS can be reflected by the cross section integration and generation optimization. The LabCut framework should be deactivated (labcut $=0$ ) if it is not necessary, because the PS application consumes additional CPU time for the integration.

The inclusion of QED photon radiation in the final-state PS (QED FSR) is controlled by the ishwfin parameter. The QED radiation is simulated in a democratic way, together with the QCD 
radiation, when ishwfin $=2$. If it is specified as ishwfin $=3$, the QED radiation is enforced and users have to give the number of photons to be generated (nphgen) and the minimum photon energy in the center-of-mass frame (ephmin) explicitly in UPINIT. The ephmin value should be equal to the minimum requirement of the photon transverse momentum.

In addition to the collinear divergences, we have to take the soft-gluon divergence into consideration in order to regularize the MEs of the processes including two partons in the final state. We apply a combined subtraction in which both divergences are simultaneously subtracted. Whereas, the PS simulations for restoring the subtracted components include the collinear components only. We need to apply a correction to PS-applied lower parton-multiplicity events in order to compensate for the alteration in the subtraction. The activation of this correction is controlled by the parameter isgcorr.

The event weights of the forced photon radiation and soft-gluon correction can be evaluated only after the PS simulations are applied. Therefore, the LabCut framework has to be activated (labcut = 1) when one of these functions is activated.

\section{2. grcpar.F}

The routines in grcpar.F are frequently called during the execution. The subroutine GRCPAR in grcpar.F controls the execution of BASES. The parameter NCALL defines the accuracy of the integration. We usually recommend that the NCALL value should be increased until the integration accuracy is reduced down to $0.2 \%$. However, it is difficult to achieve this requirement for large multiplicity processes such as diphoton + two jets. Although better integration accuracy leads to a better event generation efficiency, worse integration accuracy does not deteriorate the accuracy of event generation, unless unreasonable jumps of the integration result are observed in the iteration steps or the event weight exceeds the estimated maximum during event generation. Users should find a reasonable NCALL value for minimizing the total CPU time, although an integration accuracy of better than $1 \%$ is desired to avoid unexpected behaviors.

Another important function of the routines in grcpar.F is to determine the energy scales of the events. It is recommended to use the user-defined definition by setting ICOUP $=6$ and IFACT $=6$ in GRCPAR when the radiation matching is required. The subroutine GRCUSRSETQ is called for defining the renormalization scale GRCQ and the factorization scale GRCFAQ with this setting. However, this scheme is not sufficient to accomplish satisfactory matching because multiple definition is not allowed. We need to evaluate the energy scales for embedded non-radiative events during the subtraction, simultaneously with those for the radiative event under consideration. In addition, sometimes we may have to define the PS and subtraction energy scales independently of the renormalization and factorization scales.

The routines in the sample programs are arranged in order to fulfil these requirements. The base definition is given by the subroutine setscale according to the given set of four-momenta of interacting particles. This routine is called by GRCUSRSETQ for setting GRCQ and GRCFAQ, and it is also called by matching routines for tentatively evaluating the energy scales of non-radiative events. The routine for defining the final-state PS scale (scale_fsr) and that for defining the final-state subtraction scale (scale_fsub) are also separately provided. Although these two scales should be identical to accomplish the matching, independent routines are prepared because users may want unusual definitions for complicated studies. The PS and subtraction scales in the initial state are always identical to the factorization scale in our matching method.

The selection of generated events can be done by two subroutines, GRCUSRCUT and grclabcut. The selection in GRCUSRCUT is carried out on the basis of the hard-interaction event information before applying PS simulations, while the selection in grclabcut is based on the information after the application of PS. The latter is called before passing the cross section value to BASES/SPRING when the LabCut framework is activated by labcut $=1$. In this case, the selection condition in grclabcut is reflected by the cross section integration and event-generation optimization. In the sample programs, the event selection is completed by grclabcut and no further selection is applied. Hence, the integrated cross section of BASES can be referred to in the event mixing without any correction. We recommend users to use grclabcut for the event selection, when the selection is based on quantities that may be strongly affected by the application of PS simulations, such as the transverse momenta and production angles of photons in photon production processes. Here, 
Table 2: CPU time consumption of the external "matched diphoton + 2 jets" sample program. The time that is consumed for the cross-section integration by BASES and that for the event generation by SPRING are separately presented for each subprocess. The achieved integration accuracy is also presented. The results were obtained using 2.8-GHz CPU computers with the SLC 5 operating system.

\begin{tabular}{lccccccc}
\hline Subprocess & $a a 2 j$ & $a a 1 j$ & $a a 0 j$ & $a 2 j$ & $a 1 j$ & $q c d 2 j$ & $g g 2 a a$ \\
\hline \multirow{2}{*}{ BASES } & $95 \mathrm{~h} 25 \mathrm{~m}$ & $1 \mathrm{~h} 3 \mathrm{~m}$ & $3 \mathrm{~m} 13 \mathrm{~s}$ & $16 \mathrm{~h} 17 \mathrm{~m}$ & $50 \mathrm{~m} 18 \mathrm{~s}$ & $34 \mathrm{~h} 41 \mathrm{~m}$ & $12 \mathrm{~m} 0 \mathrm{~s}$ \\
accuracy (\%) & 0.79 & 0.17 & 0.14 & 0.37 & 0.28 & 0.19 & 0.98 \\
SPRING (s/event) & 9.1 & 0.015 & 0.0010 & 0.49 & 0.024 & 0.51 & 0.19 \\
\hline
\end{tabular}

we note that grclabcut is identical among all the subprocesses in the sample programs, while the hard-interaction generation condition is different.

The soft-gluon correction needs to be applied to lower parton-multiplicity processes in order to compensate for the alteration of the subtraction caused by the existence of soft-gluon divergences in multiple parton production processes. Although the overall activation of the correction is controlled by the isgcorr parameter in UPINIT, users may want a process-dependent control when events of multiple processes are simultaneously generated. Such a control can be achieved by using the subroutine sgcreq. This routine is called only when isgcorr $=1$.

\section{3. grappa.f}

This file contains the main program for the event generation, in which the overall event-generation control, event analyses, and I/O control are carried out. The I/O routines in GR@PPA 2.9 supports three formats of the output event file: ASCII, binary, and LHEF. The selection can be done by using the ifmt parameter.

The sample programs also have a function to add hard-interaction event information to the output events. This information is useful for checking the possible bias of the event generation condition defined in UPINIT and GRCUSRCUT to the finally selected sample. Users need to appropriately modify the programs that read the recorded events in order to utilize the added information. This function should be deactivated if such a modification cannot be done.

\section{Practical performance}

The portability of the program has been tested on various Linux distributions. The programs have been developed on Scientific Linux CERN (SLC) 5, and the scripts for building and executing the programs have been tailored so that all the functions should work properly on SLC 6 as well. The used compilers are gcc 4.1 on SLC 5 and gcc 4.8 on SLC 6 . The execution of the built-in sample programs was also tested on more recent Linux distributions: CentOS 7 (gcc 4.8), Ubuntu 16.04 (gcc 5.4), and Fedora 24 (gcc 6.3). We confirmed that we can build and execute the sample programs if external libraries are properly installed. The PYTHIA and LHAPDF libraries have to be installed by users from the source programs. Refer to the GR@PPA 2.8 manual [26] for the installation of these external libraries.

We can choose HBOOK or RBOOK as the histogramming tool in the built-in sample programs. We need to install CERNLIB libraries for using HBOOK, and ROOT libraries for using RBOOK. Although the binary distributions of ROOT are available from its official Web site and/or standard repositories of the Linux distributions, the availability of CERNLIB is limited on some platforms as the official support by CERN has already been terminated. We could not find the binary distributions of CERNLIB for CentOS 7 and Fedora 24, while it could be installed from the EPEL repository on SLC 6 and from the standard repository on Ubuntu 16.04. In the latter two cases, the installed libraries need to be linked with the "static" option. See the comments in the sample programs for more details. We provide only the HBOOK version for the external sample programs. However, if necessary, the conversion to RBOOK would be straightforward since HBOOK is used only for histogramming. 
The CPU time consumed for the execution of the external "matched diphoton +2 jets" sample program is summarized in Table 2. The test was carried out on Intel Xeon $5560(2.8 \mathrm{GHz})$ machines operated with SLC 5. The program size was approximately 4.5 MB. The virtual memory size was $20 \mathrm{MB}$, and the physical (resident) size was approximately $4 \mathrm{MB}$ including the shared memory of about $2 \mathrm{MB}$ during the execution. The CPU time consumption for the integration/optimization by BASES and that for the event generation by SPRING are separately presented in the table for each subprocess: $\gamma \gamma+2$ jets $(a a 2 j), \gamma \gamma+1$ jet $(a a 1 j), \gamma \gamma+0$ jet $(a a 0 j), \gamma+2$ jets $(a 2 j), \gamma+1$ jet $(a 1 j)$, QCD 2-jets $(q c d 2 j)$, and the loop-mediated $g g \rightarrow \gamma \gamma(g g 2 a a)$. The results are strongly dependent on the subprocess. In general, the time consumption increases as the particle multiplicity increases. Additional time is consumed for the single- $\gamma$ production processes $(a 2 j$ and $a 1 j$ ) and $q c d 2 j$, because the radiation of energetic photon(s) is required in PS.

The results for $g g 2 a a$ is markedly worse although it is a simplest two-body production process. This is because the PS energy scale is set to be very large in this subprocess in order to include hard radiation effects. The constraint on the initial hard interaction had to be significantly relaxed in order to avoid any bias to the final sample, as the kinematical alteration by PS is very large.

We carried out the test by using the built-in PDF with control parameters that had been optimized in a previous study with another PDF. Better integration accuracy can be achieved by increasing the NCALL parameter, although the integration time will increase in proportion to NCALL.

\section{Summary}

Our understanding of photon production processes in hadron collisions is still insufficient. We have explicitly shown large contributions from parton-associated processes to the direct (single) photon and diphoton (double photon) production in proton-proton collisions in our previous studies [19, 20, 21]. We release the simulation programs used in these studies as a program package, GR@PPA 2.9. The included programs consistently combine ME-based simulations for parton-associated processes up to two partons with PS simulations that reproduce the QCD multiple radiation and QED fragmentation.

The simulation method and resultant physics performance have already been described in the previous articles. We provided instructions for the installation and execution of the programs in this article. Since the previous release of GR@PPA, GR@PPA 2.8, some parameters have been newly introduced and some have been extended in order to control the new functions, namely the LabCut framework, forced photon radiation, and soft-gluon correction. In addition, programs for defining the energy scales have been modified to accomplish exact matching between the processes to be combined. The LabCut framework provides a new scheme for event selection based on the event information after the application of PS. The selection condition is reflected by the cross section integration and eventgeneration optimization.

We have confirmed that the programs can be built and executed on various Linux platforms. The practical performance of the program was presented for a sample program of the "matched diphoton +2 jets" process. The CPU time consumption is strongly dependent on the composing subprocess. In general, longer CPU time is required for larger multiplicity processes. The requirements of additional photon radiation in the PS simulation result in additional consumption of CPU time.

\section{Acknowledgments}

This work has been carried out as an activity of the NLO Working Group, a collaboration between the Japanese ATLAS group and the numerical analysis group (Minami-Tateya group) at KEK. The authors wish to acknowledge useful discussions with the members, especially S. Tsuno and J. Fujimoto of KEK, and K. Kato of Kogakuin U. 


\section{References}

[1] F. Halzen, D. M. Scott, On the Recent Observation of the Hadroproduction of Prompt Photons, Phys. Rev. D 21 (1980) 1320. doi:10.1103/PhysRevD.21.1320.

[2] J. F. Owens, Large Momentum Transfer Production of Direct Photons, Jets, and Particles, Rev. Mod. Phys. 59 (1987) 465. doi:10.1103/RevModPhys.59.465.

[3] A. Kumar, K. Ranjan, M. K. Jha, A. Bhardwaj, B. M. Sodermark, R. K. Shivpuri, Study of parton $k_{T}$ smearing effects in direct photon production at the Fermilab Tevatron, Phys. Rev. D 68 (2003) 014017. doi:10.1103/PhysRevD.68.014017.

[4] P. Aurenche, M. Fontannaz, J.-P. Guillet, E. Pilon, M. Werlen, Recent critical study of photon production in hadronic collisions, Phys. Rev. D 73 (2006) 094007. arXiv:hep-ph/0602133, doi:10.1103/PhysRevD.73.094007.

[5] V. M. Abazov, et al., Measurement of the isolated photon cross section in $p \bar{p}$ collisions at $\sqrt{s}=$ 1.96 TeV, Phys. Lett. B 639 (2006) 151, [Erratum: Phys. Lett. B 658 (2008) 285]. arXiv:hepex/0511054, doi:10.1016/j.physletb.2007.06.047, 10.1016/j.physletb.2006.04.048.

[6] T. Aaltonen, et al., Measurement of the Inclusive Isolated Prompt Photon Cross Section in $p \bar{p}$ Collisions at $\sqrt{s}=1.96 \mathrm{TeV}$ using the CDF Detector, Phys. Rev. D 80 (2009) 111106. arXiv:0910.3623, doi:10.1103/PhysRevD.80.111106.

[7] G. Aad, et al., Measurement of the inclusive isolated prompt photon cross section in $p p$ collisions at $\sqrt{s}=7 \mathrm{TeV}$ using $35 \mathrm{pb}^{-1}$ of ATLAS data, Phys. Lett. B 706 (2011) 150. arXiv:1108.0253, doi:10.1016/j.physletb.2011.11.010.

[8] S. Chatrchyan, et al., Measurement of the Differential Cross Section for Isolated Prompt Photon Production in pp Collisions at $7 \mathrm{TeV}$, Phys. Rev. D 84 (2011) 052011. arXiv:1108.2044, doi:10.1103/PhysRevD.84.052011.

[9] S. Catani, M. Fontannaz, J. Guillet, E. Pilon, Cross section of isolated prompt photons in hadron-hadron collisions, JHEP 0205 (2002) 028. arXiv:hep-ph/0204023, doi:10.1088/1126$6708 / 2002 / 05 / 028$.

[10] S. Frixione, Isolated photons in perturbative QCD, Phys. Lett. B 429 (1998) 369. arXiv:hepph/9801442, doi:10.1016/S0370-2693(98)00454-7.

[11] J. M. Campbell, R. K. Ellis, C. Williams, Direct Photon Production at Nextto-Next-to-Leading Order, Phys. Rev. Lett. 118 (2017) 222001. arXiv:1612.04333, doi:10.1103/PhysRevLett.118.222001.

[12] G. Aad, et al., Observation of a new particle in the search for the Standard Model Higgs boson with the ATLAS detector at the LHC, Phys. Lett. B 716 (2012) 1. arXiv:1207.7214, doi:10.1016/j.physletb.2012.08.020.

[13] S. Chatrchyan, et al., Observation of a new boson at a mass of $125 \mathrm{GeV}$ with the CMS experiment at the LHC, Phys. Lett. B 716 (2012) 30. arXiv:1207.7235, doi:10.1016/j.physletb.2012.08.021.

[14] T. Binoth, J. P. Guillet, E. Pilon, M. Werlen, A full next-to-leading order study of direct photon pair production in hadronic collisions, Eur. Phys. J. C 16 (2000) 311. arXiv:hep-ph/9911340, doi:10.1007/s100520050024.

[15] S. Catani, L. Cieri, D. de Florian, G. Ferrera, M. Grazzini, Diphoton production at hadron colliders: a fully-differential QCD calculation at NNLO, Phys. Rev. Lett. 108 (2012) 072001, [Erratum: Phys. Rev. Lett. 117 (2016) 089901]. arXiv:1110.2375, doi:10.1103/PhysRevLett.108.072001, 10.1103/PhysRevLett.117.089901.

[16] J. M. Campbell, R. K. Ellis, Y. Li, C. Williams, Predictions for diphoton production at the LHC through NNLO in QCD, JHEP 07 (2016) 148. arXiv:1603.02663, doi:10.1007/JHEP07(2016)148. 
[17] G. Aad, et al., Measurement of isolated-photon pair production in $p p$ collisions at $\sqrt{s}=7 \mathrm{TeV}$ with the ATLAS detector, JHEP 01 (2013) 086. arXiv:1211.1913, doi:10.1007/JHEP01(2013)086.

[18] S. Chatrchyan, et al., Measurement of differential cross sections for the production of a pair of isolated photons in $p p$ collisions at $\sqrt{s}=7$ TeV, Eur. Phys. J. C 74 (2014) 3129. arXiv:1405.7225, doi:10.1140/epjc/s10052-014-3129-3.

[19] S. Odaka, Y. Kurihara, Consistent simulation of nonresonant diphoton production at hadron collisions with a custom-made parton shower, Phys. Rev. D 85 (2012) 114022. arXiv:1203.4038, doi:10.1103/PhysRevD.85.114022.

[20] S. Odaka, Y. Kurihara, Consistent simulation of direct-photon production in hadron collisions including associated two-jet production, Mod. Phys. Lett. A 31 (2016) 1650099. arXiv:1509.04813, doi:10.1142/S0217732316500991.

[21] S. Odaka, Y. Kurihara, Consistent simulation of nonresonant diphoton production in hadron collisions including associated jet production up to two jets, Phys. Rev. D 94 (2016) 114015. arXiv:1607.00204, doi:10.1103/PhysRevD.94.114015.

[22] T. Ishikawa, et al., GRACE manual: Automatic generation of tree amplitudes in Standard Models: Version 1.0, KEK Report, KEK-92-19 (1993).

[23] F. Yuasa, et al., Automatic computation of cross-sections in HEP: Status of GRACE system, Prog. Theor. Phys. Suppl. 138 (2000) 18. arXiv:hep-ph/0007053, doi:10.1143/PTPS.138.18.

[24] S. Odaka, Y. Kurihara, Initial-state parton shower kinematics for NLO event generators, Eur. Phys. J. C 51 (2007) 867. arXiv:hep-ph/0702138, doi:10.1140/epjc/s10052-007-0342-3.

[25] S. Odaka, Simulation of $Z$ boson $p_{T}$ spectrum at Tevatron by leading-order event generators, Mod. Phys. Lett. A 25 (2010) 3047. arXiv:0907.5056, doi:10.1142/S0217732310034122.

[26] S. Odaka, Y. Kurihara, GR@PPA 2.8: Initial-state jet matching for weak boson production processes at hadron collisions, Comput. Phys. Commun. 183 (2012) 1014. arXiv:1107.4467, doi:10.1016/j.cpc.2011.12.023.

[27] T. Sjostrand, S. Mrenna, P. Z. Skands, PYTHIA 6.4 Physics and Manual, JHEP 05 (2006) 026. arXiv:hep-ph/0603175, doi:10.1088/1126-6708/2006/05/026.

[28] Y. Kurihara, et al., QCD event generators with next-to-leading order matrix-elements and parton showers, Nucl. Phys. B 654 (2003) 301. arXiv:hep-ph/0212216, doi:10.1016/S0550-3213(03)000579 .

[29] S. Hoeche, S. Schumann, F. Siegert, Hard photon production and matrix-element parton-shower merging, Phys. Rev. D 81 (2010) 034026. arXiv:0912.3501, doi:10.1103/PhysRevD.81.034026.

[30] L. D'Errico, P. Richardson, Next-to-Leading-Order Monte Carlo Simulation of Diphoton Production in Hadronic Collisions, JHEP 02 (2012) 130. arXiv:1106.3939, doi:10.1007/JHEP02(2012)130.

[31] S. Odaka, N. Watanabe, Y. Kurihara, ME-PS matching in the simulation of multi-jet production in hadron collisions using a subtraction method, PTEP 2015 (2015) 053B04. arXiv:1409.3334, doi:10.1093/ptep/ptv059.

[32] L. Bourhis, M. Fontannaz, J. Guillet, Quarks and gluon fragmentation functions into photons, Eur. Phys. J. C 2 (1998) 529. arXiv:hep-ph/9704447, doi:10.1007/s100520050158.

[33] J. Pumplin, D. R. Stump, J. Huston, H. L. Lai, P. M. Nadolsky, W. K. Tung, New generation of parton distributions with uncertainties from global QCD analysis, JHEP 07 (2002) 012. arXiv:hep-ph/0201195, doi:10.1088/1126-6708/2002/07/012.

[34] M. R. Whalley, D. Bourilkov, R. C. Group, The Les Houches accord PDFs (LHAPDF) and LHAGLUE, in: HERA and the LHC: A Workshop on the implications of HERA for LHC physics. Proceedings, Part B, 2005, pp. 575-581. arXiv:hep-ph/0508110. 
[35] J. Alwall, et al., A Standard format for Les Houches event files, Comput. Phys. Commun. 176 (2007) 300. arXiv:hep-ph/0609017, doi:10.1016/j.cpc.2006.11.010.

[36] S. Kawabata, A New Monte Carlo Event Generator for High-Energy Physics, Comput. Phys. Commun. 41 (1986) 127. doi:10.1016/0010-4655(86)90025-1.

[37] S. Kawabata, A new version of the multidimensional integration and event generation package BASES/SPRING, Comp. Phys. Commun. 88 (1995) 309. doi:10.1016/0010-4655(95)00028-E. 